\title{
Ethical issues with cognitive enhancement
}

Over the last few years I have had a small but steady number of normal students asking for stimulants such as methylphenidate (Ritalin, Concerta) to help them with their studying and exams. This may partly be because my rooms are near our local university campus.

Methylphenidate (Ritalin) was launched onto the market in 1955 and almost every year since there has been an alarmist article in the Sunday Times on the negative aspects or reactions to this drug. Myths seem to have attached themselves to these stimulants mostly on the themes of "controlling or altering the mind". If one considers that there are 2 million prescriptions written per month for Methylphenidate in the USA alone, one is going to get some stories that circulate around the morning coffee tables. It is interesting to note that until recently it had no street value and was not a drug of abuse, but now with the fashion of snorting almost everything under the kitchen sink, it has been pulled into the mixtures.

In recent years, stimulant medications have increasingly been prescribed and used to improve academic performance in normal students (called cognitive enhancement). This raises several ethical questions. Is it fair if the schoolboy or girl on methylphenidate wins the Maths prize? Should the university student on methylphenidate who gets distinctions be awarded the bursary? Should the manager on methylphenidate or other prescribed stimulants who finishes his project fastest and in the most detail get the promotion?

In addition, under the ethic of distributive justice only a small percentage of the students are able to afford these expensive drugs, so this is discriminatory on the basis of economic inequality.

There are other ethical issues. Methylphenidate is licensed to be prescribed for ADHD and narcolepsy. Prescribing it to improve academic performance in a normal subject is prescribing the drug "off-label" and not in the recommendations of the producers. We are into unknown territory here firstly because we are giving it to normal healthy subjects. It is one thing to prescribe a medication for an illness but other issues arise when giving it to normal bystanders. One runs the risk of giving side effects to healthy people. The "off-label" nature of the prescription and the potential harms (material risks) have to be explained to individual clients (they are not patients in a true sense) as well as the alternatives available.

Another question that arises is, do these stimulants actually work and improve thinking, memory, and academic performance? The evidence of efficacy is not conclusive. One needs to do a comparison with a placebo, a double expresso or some Red Bull. The effects may only be due to the general alertness, wakefulness and energy of the stimulant that allows one to concentrate and study.

Let us take a group of "normal" non-incendiary students. How do you actually chose them for a trial? Firstly, there are the baselines of cognitive functioning. There are the low performers, Mr Average in the middle, and the high flyers.

You would think that if you gave stimulants to the high flyers in the class you could improve them into Supergalactic Flyers but this is not necessarily the case; there appears to be an optimum limit and then their performances may actually deteriorate.

One of the great problems of doing research in this area of human cognition is the problem of the variability in response across and within individuals. If you do manage to find a "normal" group to do research on, some may not respond at all or very little while others may greatly benefit from the intervention. Will the low academic performers benefit the most, or will specific areas be improved while others are not improved or compromised? There may also be, in the normal group, subjects with undiagnosed ADHD or subgroups with specific genetic predispositions that react in positive or negative ways to the drugs.

The use of enhancements has been around for a longtime. Performance in sport with steroids, enhancement of physical features with cosmetics, and improvement of sexual function are all aimed at improving on "the normal".

We are now into an era of "improving on nature" and making people better than well. It is the $21^{\text {st }}$ century of Superman and Superwoman. This is now referred to as the Post-Human State.

Pharmaceutical cognitive enhancements (known as academic steroids or study drugs) are a recent unknown field of endeavour, which will raise complex ethical and moral dilemmas. Conservatives caution that it will undermine the value of human effort while others argue for its promotion to maximize human potential.

I envisage the mother of the future sending her child off to school and saying "here is your concentration pill and here is your memory pill, and don't forget your calculating pill for Maths." "Oh, and here are your vitamins." "Now remember to switch on the microchip that we had implanted in your brain last year."

I hope she does not forget the child's lunch.

Chris Ellis is a family physician from Pietermaritzburg, KwaZulu-Natal

Email: cristobalellis@gmail.com 